$10 / 26.90950$

UCID-20892

\title{
High-Temperature, Radiation-Tolerant Electronics for the MMW Space Reactor Program
}

\author{
J. H. Yee \\ W. J. Orvis \\ C. McConaghy \\ D. R. Ciarlo
}

October 17, 1986

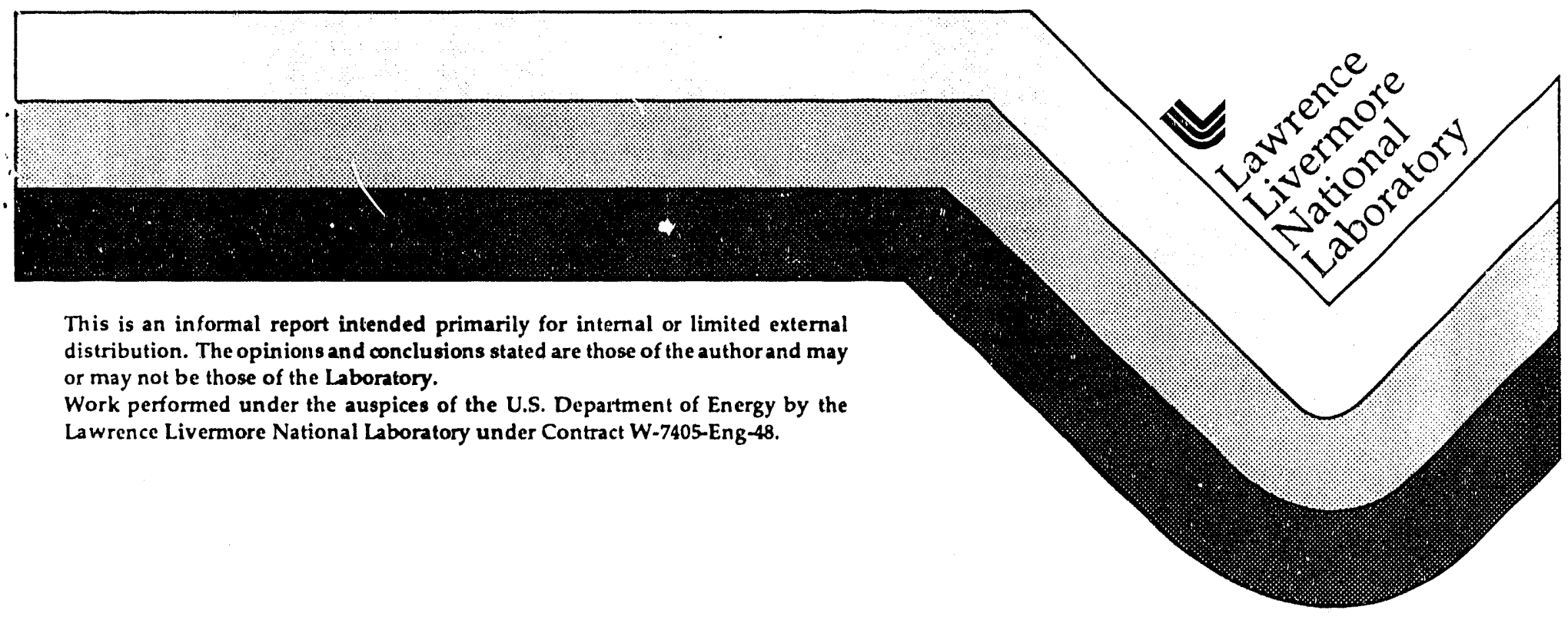


FOREWORD

This work was performed as part of the Lawrence Livermore National Laboratory effort in support of the Multi-megawatt Space Reactor Program of the Office of Space Reactor Projects, Department of Energy.

\author{
Carl E. Walter \\ MMW Project Leader \\ Nuclear Systems Safety Program
}

\title{
DISCLAIMER
}

This report was prepared as an account of work sponsored by an agency of the United States Government. Neither the United States Government nor any agency thereof, nor any of their employees, makes any warranty, express or implied, or assumes any legal liability or responsibility for the accuracy, completeness, or usefulness of any information, apparatus, product, or process disclosed, or represents that its use would not infringe privately owned rights. Refer. ence herein to any specific commercial product, process, or service by trade name, trademark, manufacturer, or otherwise does not necessarily constitute or imply its endorsement, recommendation, or favoring by the United States Government or any agency thereof. The views and opinions of authors expressed herein do not necessarily state or reflect those of the United States Government or any agency thereof.

\section{MASTER}


TABLE OF CONTENTS

Title Page

1. Summary $\ldots \ldots \ldots \ldots \ldots \ldots \ldots \ldots \ldots \ldots \ldots \ldots \ldots \ldots \ldots \ldots \ldots \ldots \ldots \ldots$

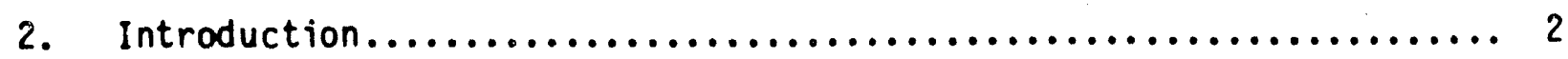

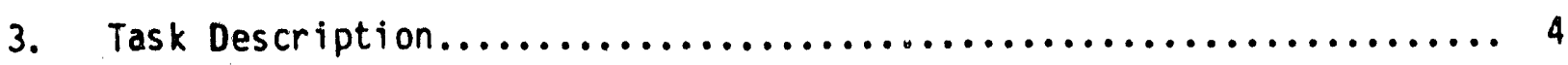

4. Results....................................... 4

5. Review of the Current Status of Miniature Vacuum Tubes........... 5

6. Proposed Process - The LLML Miniature Vacuum Triode.............. 9

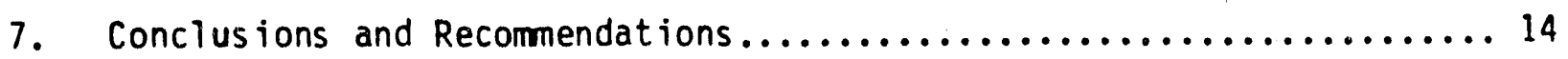

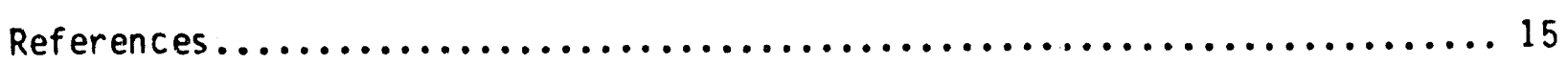




\section{SUMMAPY}

One of the objectives of the MMW space reactor program is to determine, within the next five years, what types of power electronic devices would be suitable for MMW space power applications. Suitable devices must be able to withstand high temperatures and high radiation fields. After investigating the literature on solid state device and miniature vacuum tube technologies, we have concluded that the miniature vacuum tube technology is, currentiy, the most promising. The main reason for choosing this technology, is because miniature vacuum tubes can operate at very high temperatures $(775 \mathrm{~K}$ or potentially higher) and are tolerant to very high neutron fluence and gamma dose (see Table 1). (1-5)

Although there are still problems to be solved before miniature vacuum tubes can be used, the time required for their development will be much shorter than the five year period required by the MW space reactor program.

TABLE 1. THERMIONIC INTEGRATED CIRCUIT OPERATION IN HOSTILE ENVIRONMENTS (Values tested to, no damage found)

High Temperature

Total Radiation Dose

Radiation Dose Rate

Projected Upset Level
$775 \mathrm{~K}$ for over 13,000 hours

1017 neutrons $/ \mathrm{cm}^{2}$

$2.5 \times 10^{8} \operatorname{Rad}(\mathrm{Si})$ (gamma)

$4.5 \times 10^{10} \operatorname{Rad}(\mathrm{Si}) / \mathrm{s}$ (gamma)

$2 \times 10^{8} \operatorname{Rad}(\mathrm{Si}) / \mathrm{s}$ (proton)

$1.4 \times 10^{16} \mathrm{n} / \mathrm{cm}^{2}-\mathrm{s}$

$4 \times 10^{11} \operatorname{Rad}(\mathrm{Si}) / \mathrm{s}$ (gamma) 


\section{INTRODUCTI ON}

One of the objectives of the space reactor program is to determine, within the next five years, the best power electronic devices that can be operated at very high temperatures and high radiation environments. The radiation enviroment will have a neutron dose of at least $10^{13} \mathrm{n} / \mathrm{cm}^{2}$ and a gamma dose of at least $5 \times 10^{5} \operatorname{Rad}(\mathrm{Si})$. The thermal environment will be as high as possible, in order to enhance radiation cooling of the devices. In order to meet this objective, we must either find a new solid state material or a new device technology to replace current silicon device technology. This is because silicon power devices (i.e., SCRs, Rectifiers) $(6,7)$ will be seriously degraded in the environment described above. For example, the gain of transistors has been shown to be seriously degraded with increasing neutron dose. Since SCRs are made with the same technology, they are expected to be degraded in the same manner.

For weight considerations, it is desirable to operate the electronic circuits at as high a temperature as is practical. This would reduce the amount of thermal radiator necessary to cool the devices, and hence, reduce the total mass that must be boosted into orbit. However, high temperatures will degrade or destroy the operation of most solid state devices. Silicon devices have a maximum operating temperature of $600 \mathrm{~K}$, with most devices losing their junction properties around $550 \mathrm{~K} .{ }^{(6)}$ This 10 ss of junction properties is caused by the increase of the intrinsic carrier population with temperature. When this intrinsic carrier population approaches the doping density in the material, the junction will effectively disappear. 
One way of hardening solid state devices to heat and radiation is to build the devices from large, direct band gap materials. This is because larger band gap materials are less sensitive to heat and direct band gap materials are less sensitive to radiation. Decreasing the thickness of a device or decreasing the lifetime of the minority carriers will also enhance its radiation tolerance, but will not help its themal sensitivity. Although there are many large, band-gap materials that could be used to replace silicon, however, most of them (other than GaAs) are not well characterized as to their semiconductor device properties. We do not know how to dope them properly so that we can make transistor devices with them. Table 2 shows some of the large band-gap materials. Although SiC and Diamond are promising candidates, our experience from the investigation of new materials for solar cells indicates that considerable effort would be required for their development. We estimate that it will probably take at least seven years of research and development before one learns how to make transistor devices from new materials. Therefore, to start an extensive experimental development program for these materials would be quite expensive and would take more than the allotted five years.

TABLE 2. POSSIBLE HIGH TEMPERATURE SEMICONDUCTORS( $(6)$

\begin{tabular}{|c|c|c|c|c|c|}
\hline & GaAs & GaP & BSiC & $6 \mathrm{H} \mathrm{SiC}$ & Diamond \\
\hline Band gap & $1.4 \mathrm{eV}$ & $2.2 \mathrm{eV}$ & $2.3 \mathrm{eV}$ & $2.9 \mathrm{eV}$ & $5.5 \mathrm{eV}$ \\
\hline Max operating & $725 \mathrm{~K}$ & $1150 \mathrm{~K}$ & $1200 \mathrm{~K}$ & $1500 \mathrm{~K}$ & $1375 \mathrm{~K}$ \\
\hline
\end{tabular}


A radical departure from solid state device technology is the integrated vacuum device technology. Basically, these are miniature vacuum tubes created on a substrate using semiconductor fabrication techniques. $(1-5,8-11)$ since a vacuum can not be damaged by radiation, and the operation of these devices does not depend on the band-gap of the material, they can operate at high temperature. Hence, they are very promising for the application. This led us to propose (12) that miniature vacuum technology be evaluated and possibly developed for the MMW Space Reactor Program.

\section{TASK DESCRIPTION}

The objective of this task is to review current power electronic technology to determine what material and technology would be practical and would meet the temperature and radiation requirements of the MMW Space Reactor Program within the next five years.

our approach, has been to perform a literature search to identify promising materials and technologies. As a result we have identified that miniature vacuum tubes are a most promising technology. We are now ready to start a feasibility study for its use in the MMW Space Reactor Program.

\section{RESULTS}

Two basic approaches currently exist for fabricating small structure cathodes. The first technique utilizes a mixture of barium-strontium-calcium carbonate powder and negative photoresist. This mixture is patterned to form thermionic cathodes on a sapphire substrate. The second technique utilizes 1.5 micron diameter molybdenum cones with tips that act as field emitting 
cathodes. Currently only the first technique has been applied at Los Alamos National Laboratory to fabricating more than one triode on a given substrate. It is necessary to have multiple devices on a substrate if useful circuits are to be built with this technology.

\section{REVIEW OF THE CURRENT STATUS OF MINIATURE VACUUM TUBES}

There are two main approaches to miniature vacuum tubes. One is the thermionic miniature vacuum tube which has been going on at Los Alamos National Laboratory since 1979.(1-5) Here, the effort is concentrated on the triode configurations. Two geometries have been explored, one in which source, gate, and drain (i.e., cathode, grid, and anode) all lie on the same sapphire substrate, and one in which the anode faces the cathode. One of these is shown in Fig. 1. (5) It consists of an anode, a grid, and cathode. As in a standard triode, the cathode emits electrons, the anode collects electrons, and the grid provides gain by modulating the electron flow. The process that fabricates these miniature vacuum tubes consists of four masking steps to photolithograpically del ineate thin films of refractory metals and cathode material deposited on insulating substrates.(2-4) Small scale integration for flip-flop circuits and programable power supplies has been demonstrated. The pace of advance in this program suggests that it does not face fundamental difficulties. The entire circuit runs at cathode temperature, for which the refractory materials technologies seem to be tractable. Work is now proceeding on a 3-D circuit approach in which these structures are stacked in a single hot package. The potential applications are to high temperature operation and to operation in intense radiation environments. 


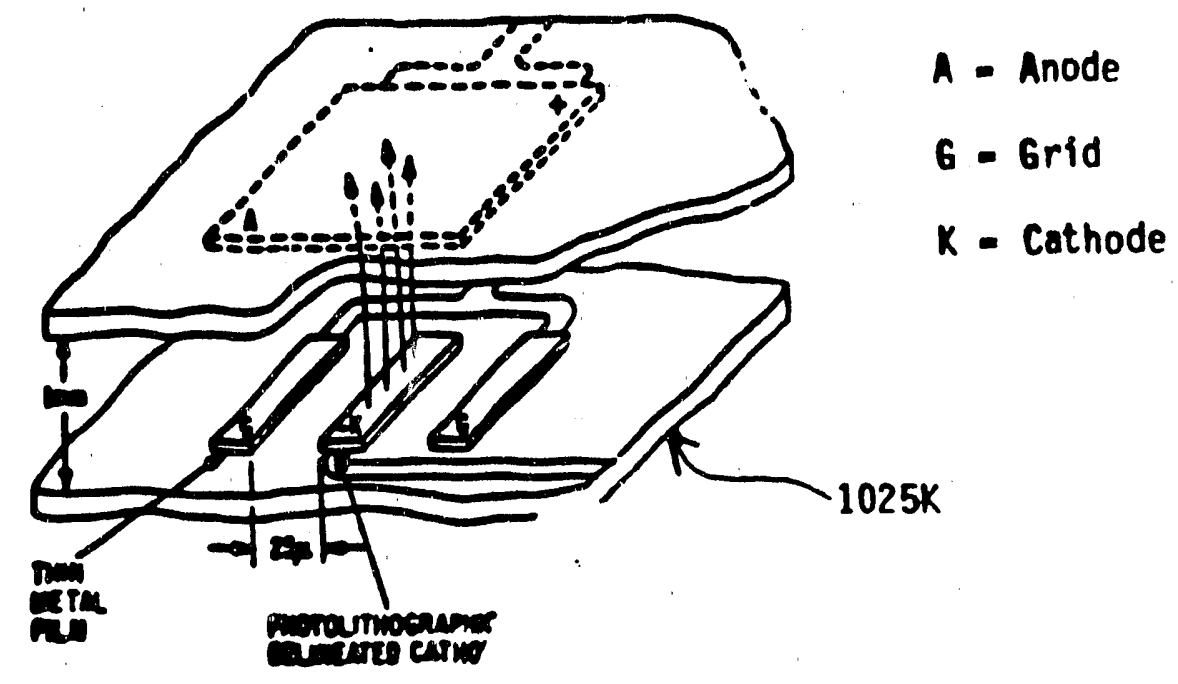

Figure 1. Structure of a miniature thermionic triode.

There appears at present to be a limitation in the density of devices on a given substrate based on Los Alamos' approach. The thermionic cathode approach is 1 imited by the thermionic cathode material which has crystals on the order of 1 micron in size. Consequently cathode to grid spacing below about 5 microns does not appear feasible. This is at least a factor of five greater than dimensions currently used in integrated circuit production.

The thermionic cathode circuits also suffer from leakage current and spurious emission problems. The leakage current problem appears to be very important in influencing the level of integration. The grid-cathode leakage can be as low as $100 \mathrm{k} \Omega$. If this leakage path exists, higher cathode currents (ten times the leakage current) must be utilized for circuits to function. The leakage is primarily caused by the utilization of negative photoresist at 
high temperature $(1175 \mathrm{~K})$ during the activation of the cathode. Photoresist is basically a cyclized rubber derivative and was never intended for high temperature operation. Photores ist processes in integrated circuit fabrication are limited to a maximum temperature of about $475 \mathrm{~K}$. In spite of the fact photoresist was not designed for high temperature work, Los Alamos has shown that through careful processing and bakeout, leakage levels can be reduced. In addition to the leakage paths caused by the processing of the thermionic cathodes, spurious emission can occur from any of the metal surfaces if the cathode material deposits on them during activation. This problem is particularly bad, when platinum is utilized since the platinum electrodes act as efficient spurious cathodes.

In summary, thermionic cathodes at present seem to have a fundamental limitation toward high levels of integration. The cathodes first can only be patterned to a minimum dimension of about 5 microns due to their material limitation. Secondly, thermionic cathode processing causes leakage currents and spurious emission paths which require larger devices (more cathode current) to swamp out the leakage currents.

Neighboring devices on a single substrate tend to interact. (1-5) This interaction can be in one of two forms. First electrons emitted from the cathode of one device may not only be collected by that device's anode but also by the electrodes of adjacent devices. The second form of interaction is due to the potentials on the electrodes in adjacent devices modifying the current flow in their neighbor. This is analogous to having parasitic grids.

Some form of isolation must be utilized between neighboring devices in order to fabricate useful circuits. Currently isolation is achieved by 
inserting electrodes at zero potential. Although these electrodes do help isolate neighboring devices, they require additional substrate real estate to implement which in turn decreases the level of integration.

The current fabrication technique for connecting the plate substrate to the cathode substrate, utilizes $1.25 \mathrm{~mm}$ platinum strips to set the cathode to anode spacing. This spacing is far from the state of the art in terms of present day integrated circuit fabrication. Although integrated circuit lithography has been utilized in cathode and grid formation, it has not been applied to the plate separation.

In spite of the fact that much work has been done at Los Alamos on miniature thermionic vacuum tubes, most of their work has been developed for small signal linear or power digital devices, not as high current or power devices. (4) Therefore, a complete evaluation for high power application would be required before one finds out how these miniature tubes would perform as power devices.

A more long range and more difficult approach has been taken at SRI for making the grid-cathode structure. $(8,11)$ This approach is based on field enitters instead of hot cathodes. The advantages of this field emitter approach are that individual devices can be fabricated at 1 um sizes or below, and that the electrons are emitted into fields of $10^{7} \mathrm{~V} / \mathrm{cm}$, so that transit times of $100 \mathrm{fs}$ are possible. Another advantage will accrue if the very high current densities of quantum (Fowler-Nordheim) tunnelling can be obtained. That is, the more abrupt nature of the tunnelling characteristic compared to the space charge characteristic shered by bipolars, and thermionic triodes will mean a higher conductance gm. 
The SRI structures are arrays of refractory metal emitters formed into sharp points by evaporation into micron or submicron holes which are formed 1ithographically. Refractory metal grids are deposited on $\mathrm{SiO}_{2}$ which insulates them from the field emitters. Vertical dimensions of $1 \mu m$ with lateral separations of 3-5 $\mu \mathrm{m}$ have been demonstrated using an ingenious selfaligned process.

In spite of the fact that SRI has successfully developed the high density grid-cathode structure, they have not used them for the development of the miniature vacuum tubes. Their work is developed primarily for the display purpose. (10)

A somewhat different approach has been taken in a newer program at NRL. The solid state processing methods used here include the e-beam lithograph and orientation-dependent-etching, to form integrally gridded sharp silicon and metal pyramids. The integrally gridded NRL device follows the Fowler-Nordheim tunnelling characteristic over miany orders of magnitudes of current. Figure 2 shows the concept of a FET-like vacuum triode, in which dimensions and parasitic capacitances are quite similar to FETS. $(8,9)$

\section{PROPOSED PROCESS - THE LLM MINIATURE VACUUM TRIODE}

The plate separation if made very small could give much smaller overall size devices. In addition the fields would be higher and the electrons would tend less to interfere with adjacent devices. Recent advances in micromachined cavities have shown cavity construction with micron dimensions. (13) We propose to utilize these same fabrication techniques to construct triodes with a plate to cathode separation of about $1 \mu \mathrm{m}$ rather than 


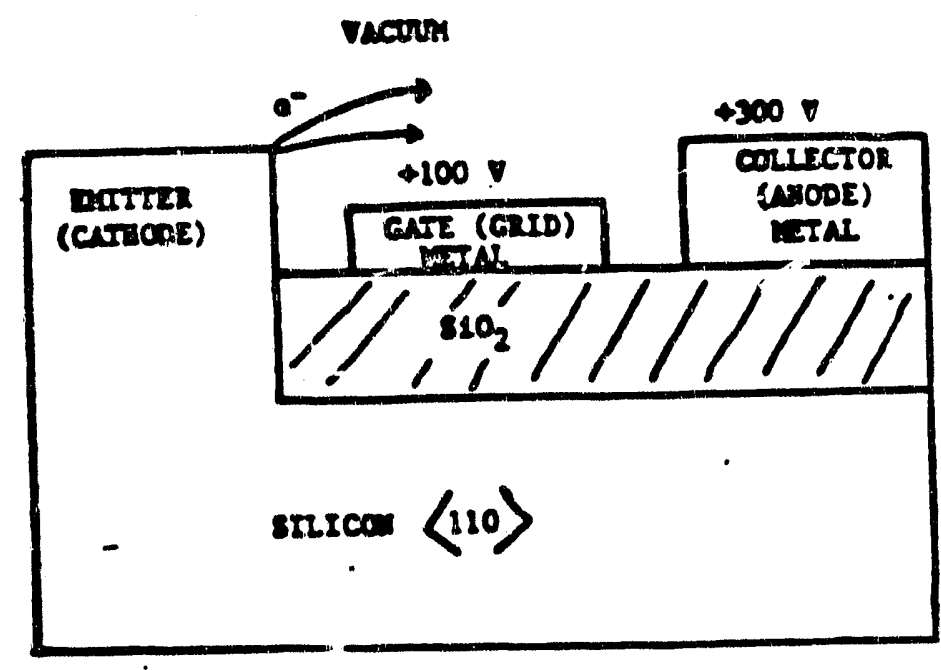

Figure 2. Concept of a FET-like vacuum field emitter triode. 
the current $1 \mathrm{~mm}$ spacing. In addition to the micromachined cavities, the use of field emitting cathodes is proposed to eliminate some of the previously mentioned problems of patterning and contamination associated with thermionic cathodes.

The following process is proposed as something we believe could be fabricated within the Solid State Devices Group at LLNL. The field emitting cathodes are similar in nature to those built at SRI International. Figure 3 shows the processing sequence.

Step 1. A sapphire substrate supports the subsequent 1ayers of material.

Step 2. Molybdenum is deposited which will act as the cathode interconnect metal.

Step 3. The molybdenum is covered with about 1.5 microns of LPC.VD silicon dioxide.

Step 4. The $\mathrm{SiO}_{2}$ is covered with molybdenum which will act as the grid.

Step 5. A 1.5 micron diameter hole is patterned in the moiybdenum, where a triode is desired. This patterning would be done with plasma etching. Multiple holes or possibly a slot hole could be utilized to fabricate different $\mathrm{Gm}$ devices.

Step 6. The $\mathrm{SiO}_{2}$ under each hole would be chemically etched with HF acid. This etch would undercut and form an overhang in the molybdenum grid.

Step 7. Molybdellum would be evaporated through the holes to form cones (by virtue of shadowing) beneath the surface of the molybdenum grid. The cones could be 1.5 microns at the base and come to a point at 
Step 1

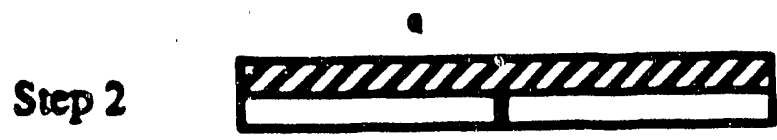

Step 3

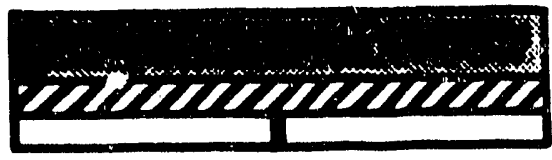

Step 4

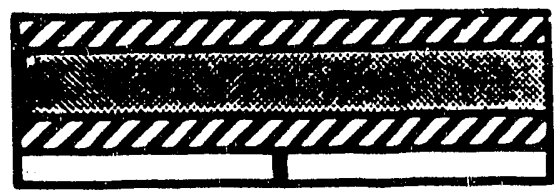

Step 5

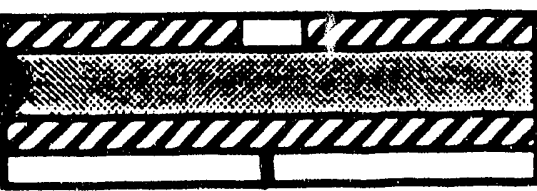

Siep 6

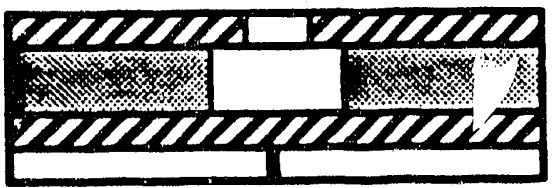

Step 7

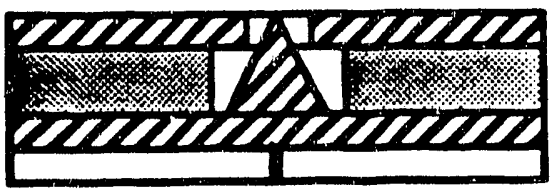

Step 8

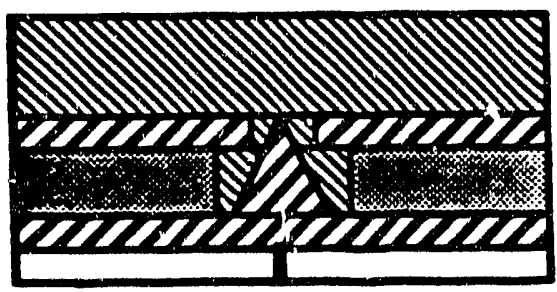

Step 9

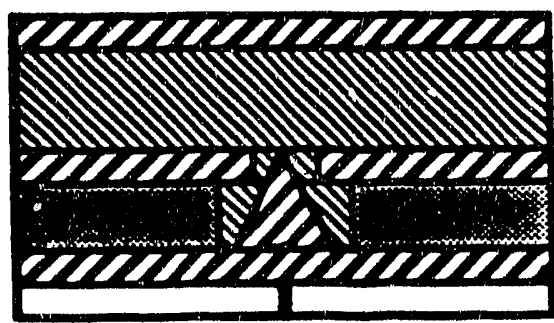

Step 10

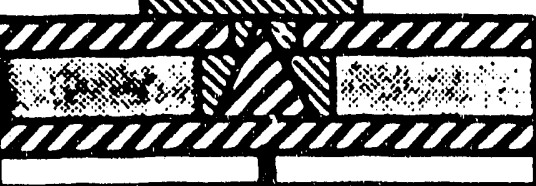

Step 11

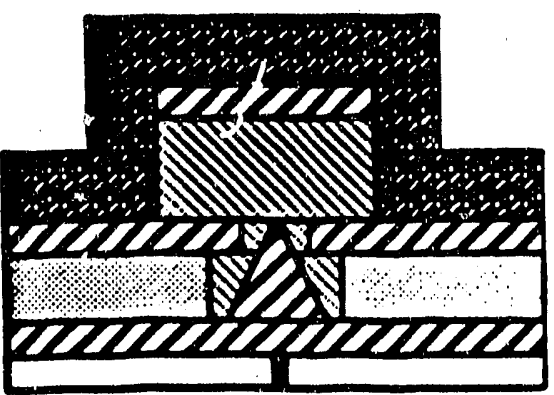

Step 12

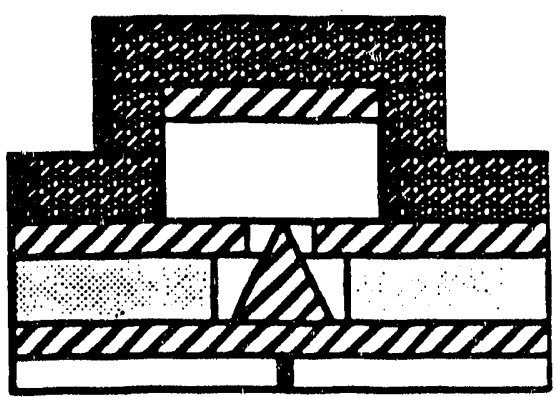

D Sapphire Substrate

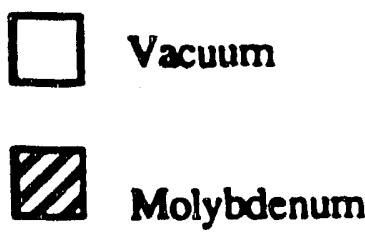

$\mathrm{SiO}_{2}$

PolySilicon

$\mathrm{Si}_{3} \mathrm{~N}_{4}$

Figure 3. Proposed Microcavity Triode Process 
the surface of the molybdenum grid. These points would be the field emitters.

Step 8. The entire substrate would be "covered" with polysilicon. The height of this polysilicon should be at least 1 to 2 microns and will determine the cathode anode spacing.

Step 9. The polysilicon is "covered" with molybdenum which will serve as the anode.

Step 10. The molybdenum is patterned to form anodes as well as anode interconnects.

Step 10a. The polysilicon underneath the anodes is patterned to form the triode cavity.

Step 11. The entire surface is covered with silicon nitride which acts as the top of the cavity.

Step 12. The polysilicon is etched with $\mathrm{KOH}$ which does not attack any of the metals, nitrode, oxide, or sapphire that are already present. The $\mathrm{KOH}$ would flow into the cavities via the ends which would be left open for etching as well as vacuum pump down.

The devices could be placed very close to one another because of the higher fields confining the electrons paths as well as minimization of electrostatic coupling from adjacent devices. The field emitter has been chosen as the cathode in this approach mostly to minimize some of the previously mentioned problems of thermionic emitters. Various gm devices could be fabricated by changing the holes for cathode formation to slots and essentially increasing cathode area. 
The two main advantages of this technology are: one, a decrease in cathode to anode spacing of between 500 to 1000 over current technology with a corresponding increase in the level of integration, and second, a truly integrated structure with no mechanical fabrication steps.

\section{CONCLUSIONS AND RECOMMENDATIONS}

After investigating the literature on power electronic device materials and technologies, we have concluded that the miniature vacuum tube technology is, currently, the most promising. The main reason for choosing this technology, is because miniature vacuum tubes can operate at very high temperatures $(775 \mathrm{~K}$ or potentially higher) and are tolerant to neutron fluence and gamma dose far beyond that required for the Space Reactor Program. Though there are a number of development problems, we believe that they can be solved well within the allowed five year period.

During the next year if our work continues to be funded, we will begin to investigate the electrical properties of our proposed miniature vacuum tubes using our 2-D particle codes. Hopefully, these investigations will enable us to determine the most promising design configurations for the miniature devices that could be used in the MMW Program. We should also fabricate a small number of devices using our solid state fabrication and miromachining techniques. These devices would be used to generate experimental data to validate the model results, and to verify the appropriateness of miniature vacuum tube technology for the MMW Space Reactor Program.

We believe that considerable progress could be made during FY87 with a budget of $\$ 200 \mathrm{~K}$. About $1 / 3$ of the effort would be applied to modeling with the remainder to fabrication of devices and measurement of their performance. 
REFERENCES

1. Lynn, D. L., et al., "Thermionic Integrated Circuits: Electronics for Hostile Environments," IEEE Transactions on Nuclear Science, Vol. NS-32, No. 6, December 1985.

2. McCormick, J. Byron, et al., "Development of Integrated Thermionic Circuits for High-Temperature Applications," High Temperature Electronics Conference," March 25-27, 1981, Tucson, AZ.

3. McCormick, J. Byron, et al., "Development of Integrated Thermionic Circuits for High-Temperature Applications," IEEE Trans. on Industrial Electronic, Vol. IE-29, No. 2, May 1981.

4. Private Communication with Dr. D. K. Lynn of Los A1amos, October 14, 1986.

5. Lynn, D. K., et al., "Progress in Radiation Immune Thermionic Integrated Circuits," LA-10466-MS, UCP-38, August 1985.

6. Sze, S. M., Physics of Semiconductor Devices, 1969.

7. Kabat, M., Power Semiconductors, 1984. 
8. R. Greene, H. Gray and G. Campisi, IEDM 85-173.

9. Private Communication with Drs. R. Greene and H. Gray of NRL.

10. Private Communication with Dr. C. A. Spindt of SRI, October 13, 1986.

11. Spindt, Charles A., et al., "Ficld Emission Cathode Array Development for High-Current-Density Applications," App. of Surf. Science $16(1983), 268-276$.

12. This work was started after a personal communication with Drs. Frank Thome and Nestor Ortiz of Sandia Laboratory, June, 1986.

13. H. Gucke1, D. W. Burns, "A Technology for Integrated Transducers," 1985 Conference on Solid-State Sensors and Actuators. 

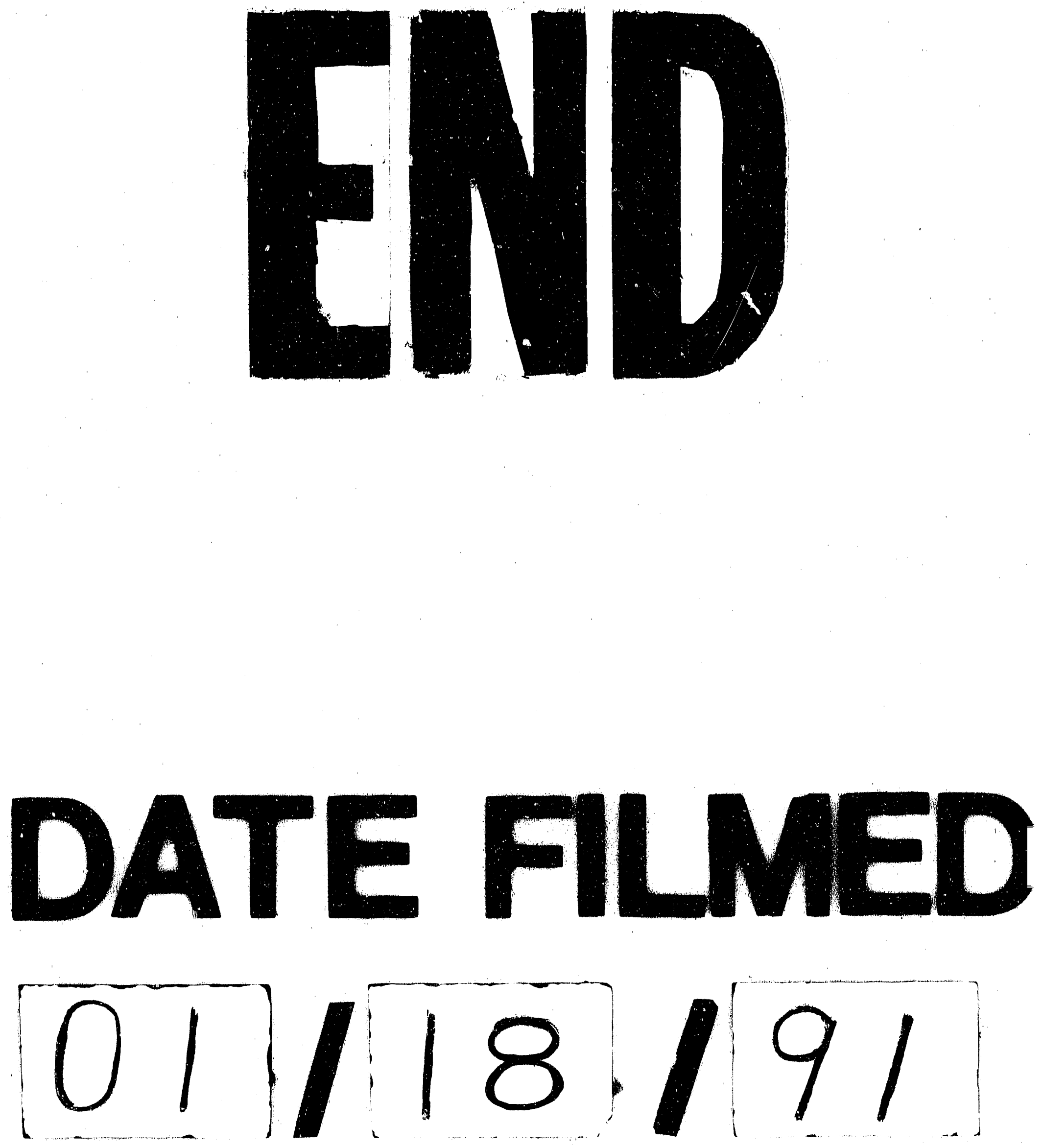
